\title{
The Role of a History of Coronary Heart Disease among Second Degree Relatives for Predicting Coronary Artery Disease
}

\author{
Saeed Alipour Parsa ${ }^{1}$, Farzam Saemifar ${ }^{1,}$, Isa Khaheshi ${ }^{1}$, \\ Mohammadreza Naderian ${ }^{2,3}$
}

${ }^{1}$ Cardiovascular Research Center, Shahid Beheshti University of Medical Sciences, Tehran, Iran

${ }^{2}$ Non-Communicable Diseases Research Center, Endocrinology and Metabolism Population Sciences Institute, Tehran University of Medical Sciences, Tehran, Iran ${ }^{3}$ Students' Scientific Research Center (SSRC), Tehran University of Medical Sciences, Tehran, Iran

* Corresponding author: Farzam Saemifar, Cardiovascular Research Center, Shahid Beheshti University of Medical Sciences, Tehran, Iran. Tel: +98-9111493114, E-mail:

DOI: $10.20286 /$ ijcp-010202 xnm59@yahoo.com

\section{Submited: 05.30.2016 \\ Accepted: 06.22.2016 \\ Keywords: \\ Risk Factors \\ Coronary Artery Disease \\ Family Relations \\ History}

(C) 2016. International Journal of Cardiovascular Practice.

\begin{abstract}
Introduction: The history of atherosclerotic disease among second degree family members of patients as a risk factor has not been properly explained. The present study aimed to assess this role in the Iranian population.

Methods: This case-control study was performed on 500 consecutive patients, who were candidates for coronary angiography. The presence of Coronary Heart Disease (CHD) history among first and second degree relatives were determined by interviewing the participants.

Results: In total, 450 patients were shown to have $\mathrm{CHD}$ as the case group and 50 without $\mathrm{CHD}$ as the control group. Family history of $\mathrm{CHD}$ among first degree relatives was $39.1 \%$ for cases and $22.0 \%$ for the controls with a significant difference $(P=0.018)$, however the history of $\mathrm{CHD}$ among second degree relatives was not statistically different in the case group and the control group $(17.8 \%$ vs. $8.0 \%, \mathrm{P}=0.079)$. In total, 80 patients had CHD with simultaneous history of disease among their second degree relatives, while 370 with CHD had no history of disease among their second degree relatives. Our study could not find a significant difference between the two CHD groups with and without history of disease among second degree relatives in terms of cardiovascular risk profile.

Conclusions: Despite the powerful effect of the presence of family history of CHD in first degree relatives on risk of CHD and its severity, the presence of this history among second degree relatives cannot predict the risk for $\mathrm{CHD}$.
\end{abstract}

\section{INTRODUCTION}

Coronary Heart Disease (CHD) is the most common cause of death in the world and a multi-factor disease influenced by several genetic and environmental factors. Several studies have shown that a family history of ischemic heart disease is a very important predictor for CHD that can estimate the increased risk for coronary heart disease and thus can be useful for primary prevention of the disease [1-4]. Many studies have been conducted on family history of ischemic heart disease as a main determinant for CHD. In fact, family history reflects the involvement of both genetic and ecological factors that affect the metabolic profile as well as lifestyle of families and individuals, simultaneously $[5,6]$. By definition, a positive family history of CHD refers only to the presence of disease among first-degree relatives $[7,8]$; however, a his- tory of atherosclerotic disease among second degree family members of the patients, as a risk factor, has not been properly explained. Because of limited available studies on the role of positive family history of $\mathrm{CHD}$ among second degree relatives, as a risk profile for disease, the present study aimed to assess this role in the Iranian population. In other words, in this researched we aimed to determine whether the presence of family history of CHD among second degree family members of patients could predict the risk for disease in these patients.

\section{METHODS}

This case-control study was performed on 500 consecutive patients, who were candidates for coronary angiography to confirm or rule out of documented CHD defined as the pres- 
ence of at least one involved coronary vessel with more than $50 \%$ stenosis in coronary angiography report. In this regard, the patients were categorized as the case group with evident Coronary Artery Disease (CAD) and the control group without significant coronary disease. The baseline characteristics and clinical data of the subjects including the presence of cardiovascular risk profiles were collected by reviewing the recorded hospital files or interviewing the patients. Also, the presence of CHD history among first and second degree relatives was determined by interviewing the participants.

Results were presented as mean \pm Standard Deviation (SD) for quantitative variables and were summarized by absolute frequencies and percentages for categorical variables. Normality of data was analyzed using the Kolmogorov-Smirnoff test. Categorical variables were compared using the chisquare test or Fisher's exact test when more than $20 \%$ of cells with expected count of less than five were observed. Quantitative variables were also compared with $t$ test or MannWhitney U test. For the statistical analysis, the SPSS version 16.0 statistical software for windows (SPSS Inc., Chicago, IL) was used. P values of 0.05 or less were considered statistically significant. The institutional review board approved the study protocol and patients provided informed written consent.

\section{RESULTS}

In total, 450 patients with CHD were included in the case group and 50 without $\mathrm{CHD}$ made up the control group. Comparing the two groups (Table 1), indicated higher mean age as well as higher male gender frequency in the CHD group than in the control group. Regarding cardiovascular common risk factors, the prevalence of diabetes was $33.1 \%$ and $26.0 \%$ in the case and control group, respectively, with no difference between the two groups ( $\mathrm{P}=0.308)$. Also, $56.4 \%$ and $52.0 \%$ were hypertensive, $40.9 \%$ and $36.0 \%$ were dyslipidemic and $33.8 \%$ and $22.0 \%$ were smokers in the case and control group, respectively, without between-group differences. A history of previous myocardial infarction (53.3\% vs. $2.0 \%$ ), previous Coronary Artery Bypass Grafting Surgery (CABG) (19.1\% vs. $0.0 \%$ ), and previous Percutaneous Coronary Intervention
(PCI) procedures (56.0\% vs. $2.0 \%$ ) were more common in the case group compared with the control group. In electrocardiography assessment, ST-T changes were found in $72.2 \%$ of the patients in the case group and $60.0 \%$ in the control group with a slight difference $(\mathrm{P}=0.072)$, however pathologic Q wave was significantly higher in those with documented CHD (33.6\% vs. $8.0 \%, \mathrm{P}<0.001)$. Also, indications of branch blocks were more evident in the case than in the control groups (14.0\% vs. $6.0 \%)$. Echocardiography assessment also indicated higher prevalence of left ventricular dysfunction as well as higher rate of wall motion abnormality among cases when compared to the controls $(\mathrm{P}<0.001)$.

Family history of CHD among first degree relatives was found in $39.1 \%$ of the cases and $22.0 \%$ of the controls with a significant difference $(\mathrm{P}=0.018)$, however the history of $\mathrm{CHD}$ among second degree relatives was not statistically different in the case group and the control group (17.8\% vs. $8.0 \%, \mathrm{P}=0.079$ ).

In total, 80 patients had $\mathrm{CHD}$ with simultaneous history of disease among their second degree relatives, while 370 with CHD had no history of disease among their second degree relatives. Our study could not find a significant difference between the two CHD groups with and without history of disease among second degree relatives in terms of gender $(\mathrm{P}=0.587)$, diabetes mellitus $(\mathrm{P}=0.514)$, smoking $(\mathrm{P}=0.190)$, previous myocardial infarction $(\mathrm{P}=0.284)$, history of cerebrovascular disease $(\mathrm{P}=0.610)$, and history of CABG $(\mathrm{P}=0.592)$ or PCI $(\mathrm{P}=0.149)$. However, those with a history of $\mathrm{CHD}$ among their second degree relatives were younger (mean age: 56.84 years vs. 60.62 years, $P=0.005)$ with lower prevalence rates of hypertension $(42.5 \%$ vs. $52.5 \%, \mathrm{P}=0.006)$ and dyslipidemia ( $22.5 \%$ vs. $36.8 \%, \mathrm{P}=0.015)$ (Table 2 ). Also, there was no difference in the number of involved coronary vessels between the $\mathrm{CHD}$ groups with and without family history among second degree relatives $(\mathrm{P}=0.181)$. The risk for $\mathrm{CHD}$ significantly increased among patients with a family history among first degree relatives $(\mathrm{OR}=2.27,95 \% \mathrm{CI}: 1.13$ to 4.57$)$, however the presence of $\mathrm{CHD}$ among second degree relatives could not predict the increased risk for $\mathrm{CHD}$ among the patients $(\mathrm{OR}=$ $2.49,95 \% \mathrm{CI}: 0.87$ to 7.11 ).

\begin{tabular}{|lccc|}
\hline Table 1: Baseline Characteristics and Clinical Data of Study Participants & & \\
Item & CHD Group (n=450) & Non-CHD Group (n=50) & P value \\
Male gender & $282(62.7)$ & $22(44.0)$ & 0.010 \\
Age, year & $59.95 \pm 11.07$ & $51.96 \pm 12.06$ & $<0.001$ \\
Diabetes mellitus & $149(33.1)$ & $13(26.0)$ & 0.308 \\
Hypertension & $254(56.4)$ & $26(52.0)$ & 0.548 \\
Dyslipidemia & $154(40.9)$ & $18(36.0)$ & 0.504 \\
Smoking & $152(33.8)$ & $11(22.0)$ & 0.092 \\
Myocardial infarction & $240(53.3)$ & $1(2.0)$ & $<0.001$ \\
Cerebrovascular disease & $23(5.1)$ & $1(2.0)$ & 0.329 \\
Previous CABG & $86(19.1)$ & $0(0.0)$ & 0.001 \\
Previous PCI & $252(56.0)$ & $1(2.0)$ & $<0.001$ \\
Family history of CAD (first relatives) & $176(39.1)$ & $11(22.0)$ & 0.018 \\
Family history of CAD (second relatives) & $80(17.8)$ & $4(8.0)$ & 0.079 \\
\hline
\end{tabular}

CHD: coronary heart disease; CABG: coronary artery bypass grafting; PCI: percutaneous coronary intervention; CAD: coronary artery disease.

Data in table are presented as No. (\%). 
Table 2: Baseline Characteristics and Clinical Data in the Groups with and without Family History of CHD among Second Degree Relatives

$\begin{array}{cc}\text { CHD Group with History of CHD } & \text { CHD Group without History of CHD } \\ \text { Among Second Degree Relatives }(n=80) & \text { Among Second Degree Relatives }(n=370)\end{array}$

P value

\begin{tabular}{|c|c|c|c|}
\hline Male gender & $48(60.0)$ & $234(63.2)$ & 0.587 \\
\hline Age, year & $56.84 \pm 10.10$ & $60.62 \pm 11.16$ & 0.005 \\
\hline Diabetes mellitus & $24(30.0)$ & $125(33.8)$ & 0.514 \\
\hline Hypertension & $34(42.5)$ & $220(59.5)$ & 0.006 \\
\hline Smoking & $18(22.5)$ & $136(36.8)$ & 0.015 \\
\hline Smoking & $22(27.5)$ & $130(35.1)$ & 0.190 \\
\hline Myocardial infarction & $47(58.8)$ & $193(52.2)$ & 0.284 \\
\hline Cerebrovascular disease & $5(6.2)$ & $18(4.9)$ & 0.610 \\
\hline Previous CABG & $17(21.2)$ & $69(18.6)$ & 0.592 \\
\hline Previous PCI & $39(48.75)$ & $213(60.27)$ & 0.149 \\
\hline \multicolumn{2}{|c|}{ Number of involved coronaries } & & 0.181 \\
\hline None & $13(16.2)$ & $57(15.4)$ & \\
\hline Single vessel & $12(15.0)$ & $93(25.1)$ & \\
\hline Two vessels & $28(35.0)$ & $96(25.9)$ & \\
\hline Three vessels & $27(33.8)$ & $124(33.5)$ & \\
\hline
\end{tabular}

CHD: coronary heart disease; CABG: coronary artery bypass grafting; PCI: percutaneous coronary intervention

Data in table are presented as No. (\%) or Mean \pm SD.

\section{DISCUSSION}

This case-control study showed that the presence of coronary heart disease among first degree relatives predict CHD (OR $=2.27$ ) but this history in second degree relatives cannot predict the risk for $\mathrm{CHD}$.

Reviewing the literature showed no similar comprehensive research on the association between the presence of family history of $\mathrm{CHD}$ among second degree relatives and increased risk for disease. However, several studies could demonstrate the definitive role of family history of CHD among first degree relatives for predicting $\mathrm{CHD}$ and its severity and prognosis. As shown by Leander et al. [1], family history was a strong risk factor for myocardial infarction interacting with other cardiovascular risk factors. A male candidate with one or more affected parents or siblings has an increased risk of myocardial infarction by more than two times compared with no family history of coronary heart disease. In a female candidate this increase is 2.1 times. When two or more parents or siblings are affected, the adjusted odds ratio of myocardial infarction was 3.4 and 4.4 for males and females, respectively. Family history of coronary heart disease in current female smokers had a synergistic effect with high quotient between low-density lipoprotein and high-density lipoprotein cholesterol. Hawe et al. [9] showed that the hazard ratio of CHD for males with a family history of CHD was 1.73 compared to those without a family history; and this was independent of other classic risk factors. In a systematic review by Prabhakaran et al. [10], after pooling the case-control studies (involving 17,202 cases and 30,088 controls) unadjusted odds ratio of 2.03 was yielded, whereas after pooling the cohort studies (including 313,837 individuals), relative risk for future coronary heart disease of 1.60 was yielded. All published papers focused on the predictive role of family history of CHD in first degree relatives and could also show its synergistic effects when they interacted with other cardiovascular risk factors, however the role of family history in second degree relatives was not approved, which was also shown by our study. In fact, the presence of family history of CHD in second degree relatives might not have enough power to predict an increased risk for CHD. It seems that the hereditary transfer of polymorphisms related to CHD is mainly done between first degree relatives of the patients.

However, it seems that the employed small sample size and therefore partially low power of our study could potentially affect the results. Also, one of the other main limitations of the study was lower prevalence of some cardiovascular risk factors such as advanced ages, hypertension, and diabetes in the group with a family history of CHD in second degree relatives that affected the validity of our findings. In fact, this finding may rule out the synergistic effect of family history of $\mathrm{CHD}$ in second degree relatives and other risk factors.

Overall, it seems that despite the powerful effect of the presence of family history of CHD in first degree relatives on risk of $\mathrm{CHD}$ and its severity, the presence of this history among second degree relatives cannot predict the risk of $\mathrm{CHD}$. Also, the synergistic effects of this parameter with other cardiovascular risk profiles are not predicted.

\section{ACKNOWLEDGMENTS}

There were no acknowledgments for the present study.

\section{CONFLICTS OF INTEREST}

The authors declare that they have no conflicts of interest.

\section{REFERENCES}

1. Leander K, Hallqvist J, Reuterwall C, Ahlbom A, de Faire U. Family history of coronary heart disease, a strong risk factor for myocardial infarction interacting with other cardiovascular risk factors: results from the Stockholm Heart Epidemiology Program (SHEEP). Epidemiology. 2001;12(2):215- 
21. DOI: 10.1097/00001648-200103000-00014 PMID: 11246583

2. Pohjola-Sintonen S, Rissanen A, Liskola P, Luomanmaki K. Family history as a risk factor of coronary heart disease in patients under 60 years of age. Eur Heart J. 1998;19(2):235-9. DOI: 10.1053/euhj.1997.0543 PMID: 9519316

3. Anthony D, George P, Eaton CB. Cardiac risk factors: biomarkers and genetic tests to determine cardiovascular risk. FP Essent. 2014;421:115. PMID: 24936714

4. Pandey AK, Pandey S, Blaha MJ, Agatston A, Feldman T, Ozner M, et al. Family history of coronary heart disease and markers of subclinical cardiovascular disease: where do we stand? Atherosclerosis. 2013;228(2):285-94. DOI: 10.1016/j.atherosclerosis.2013.02.016 PMID: 23578356

5. Banerjee A. A review of family history of cardiovascular disease: risk factor and research tool. Int J Clin Pract. 2012;66(6):536-43. DOI: 10.1111/j.1742-1241.2012.02908.x PMID: 22607505

6. Dhiman P, Kai J, Horsfall L, Walters K, Qureshi N. Availability and quality of coronary heart disease family history in primary care medi- cal records: implications for cardiovascular risk assessment. PLoS One. 2014;9(1):e81998. DOI: 10.1371/journal.pone.0081998 PMID 24416135

7. Hasanaj Q Wilson BJ, Little J, Montazeri Z, Carroll JC, Screening CETiGi. Family history: impact on coronary heart disease risk assessment beyond guideline-defined factors. Public Health Genomics. 2013;16(5):208-14. DOI: 10.1159/000353460 PMID: 23886802

8. Akhabue E, Thiboutot J, Cheng JW, Vittorio TJ, Christodoulidis $\mathrm{G}$, Grady $\mathrm{KM}$, et al. New and emerging risk factors for coronary heart disease. Am J Med Sci. 2014;347(2):151-8. DOI: 10.1097/ MAJ.0b013e31828aab45 PMID: 23644407

9. Hawe E, Talmud PJ, Miller GJ, Humphries SE, Second Northwick Park Heart S. Family history is a coronary heart disease risk factor in the Second Northwick Park Heart Study. Ann Hum Genet. 2003;67(Pt 2):97 106. DOI:10.1046/j.1469-1809.2003.00017.x PMID: 12675686

10. Prabhakaran D, Jeemon P. Should your family history of coronary heart disease scare you? Mt Sinai J Med. 2012;79(6):721-32. DOI: 10.1002/ msj.21348 PMID: 23239210 